\title{
Lorenz Chaos in Optically Pumped Lasers
}

\author{
R. Vilaseca ${ }^{1}$ and R. Corbalán ${ }^{2}$ \\ 1 Universitat de València, Spain \\ 2Universitat Autònoma de Barcelona, Spain
}

\begin{abstract}
There may exist universal features that make a system obey the Lorenz model for deterministic chaos because the dynamic behaviour observed in an optically pumped laser, which is of the type predicted by the model, is only weakly dependent on certain parameters.
\end{abstract}

Research over over the last eight years shows that lasers are among the most versatile physical systems for studying the dynamic behaviour of nonlinear dissipative systems where motion never becomes stable or periodic. The wide variety of laser classes, the ease with which several laser parameters can be changed and the rapidity of the internal relaxation processes have allowed the observation of a rich variety of unstable phenomena [1].

We review here recent studies of one particular type of phenomenon, namely the Lorenz behaviour of optically pumped lasers which represents the first experimental observation of a behaviour of the type predicted by the Lorenz model for deterministic chaos in nonlinear dissipative systems [2]. It is shown that Lorenz features show some universality in that they are only weakly dependent on certain variables in the model, even though the variations entail a large increase in the number of degrees of freedom. Readers are referred to the literature for other examples demonstrating the versatility of lasers for studying dynamic systems as, for example, the observation of Shilnikov dynamics in $\mathrm{CO}_{2}$ lasers with feedback or with a saturable absorber [3] and the prediction and observation of spatial structures and optical vortices in multimode lasers [4].

\section{The Lorenz Model}

In 1963, E.N. Lorenz, who was interested in the atmosphere, established a simple model for analysing fluid dynamics

\footnotetext{
Ramon Vilaseca is Professor of Optics at the Universitat Autònoma de Barcelona and has been with the Departament Interuniversitari d'Optica, Facultat de Fisica, Universitat de València, Dr. Molnar, 50, E-46100 Burjassot, Spain, since 1985. He obtained his Ph.D. in physics from the Universitat de Barcelona in 1976.
}

Ramon Corbalán has been Professor of Optics at the Universitat Autònoma de Barcelona since 1975. He received his Ph.D. in physics from the Universidad de Zaragoza in 1972.

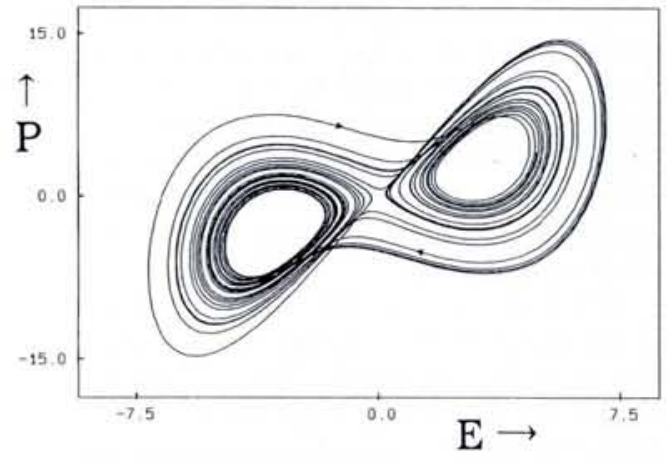

- Chaotic behaviour predicted by the Lorenz model. (a, upper) A trajectory in the phase space captured by the chaotic attractor: two symmetric stationary solutions (fixed points) lying inside the "holes" of the attractor also exist, but they are unstable for a wide range of values of the parameter $r$. (b, lower) Time evolution of $\chi^{2}$, which in the case of a laser represents the field intensity $E^{2}$ (see text). A series of peaks of increasing size are apparent, which correspond to the spiral motion in Fig. 1a (parameter values: $\sigma=1.4, b=$ $0.28, r=40$ ). For a gas laser the unit of time is of the order of $1 \mu \mathrm{s}$.

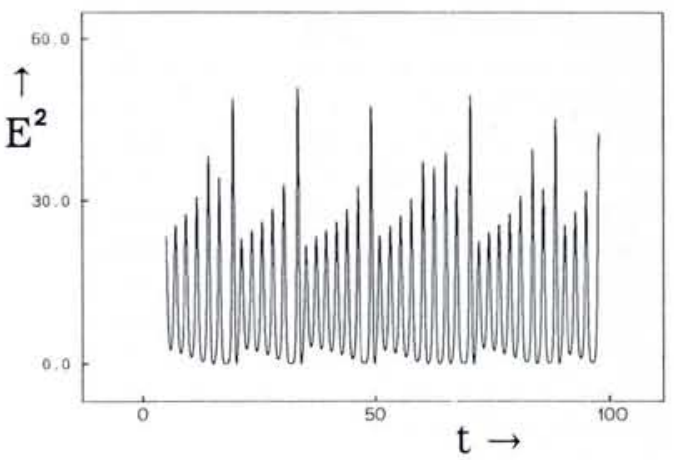

in the Rayleigh-Bénard convection problem involving the spontaneous formation of "rolls" in a horizontal fluid layer subjected to a vertical temperature gradient. Through a drastic truncation of complex fluid equations, he derived a set of only three equations which could be studied numerically:

$$
\dot{x}=\sigma(y-x) ; \dot{y}=x(r-z)-y ; \dot{z}=x y-b z
$$

where $x, y$ and $z$ are the variables describing the fluid's state ( $x$ is related to the velocity field and $y$ and $z$ to the temperature field) and $\sigma, r$ and $b$ are parameters (in particular, $\sigma$ and $r$ are related to the Prandtl and Rayleigh numbers, respectively).

These equations are quite simple in the sense that only first-order time derivatives are involved and only two nonlinear terms, namely the second-degree terms $-x z$ and $x y$, are present. In spite of this, Lorenz found that for a wide range of parameter values and initial conditions the evolution of the system never becomes stable or periodic. Owing to the presence of dissipative terms, i.e., the terms $-\sigma x,-y$ and $-b z$, the trajectory in the phase space is "attracted" towards a definite region called an attractor, remains forever in it (Fig. 1) and never shows periodic behaviour. "Motion" within the attractor is erratic since it consists of growing spirals around one fixed point, interrupted at random intervals by a jump to a symmetric fixed point. An (arbitrarily) small change in the initial conditions leads in the long term to a large divergence of the trajectories so that they become uncorrelated.

Motion characterised by this extreme sensitivity to the initial conditions is said to be chaotic. As this type of motion appeared in spite of the limited number of degrees of freedom of the system and the absence of noise terms in the equations, a new type of chaos, namely deterministic chaos had been discovered.

Since then, the Lorenz model has been considered as a paradigm of deterministic chaotic behaviour and is still widely investigated in theoretical studies of nonlinear dynamics in dissipative systems. A rich variety of dynamical features has been 
found, in particular, local bifurcations, homoclinic explosions, windows of periodic motion within chaos, crises, symmetry breaking, generalised bistability and hysteresis, and period-doubling, and intermittency roads to chaos [5], some of which are defined in the box.

\section{The Lorenz-Haken Laser Model}

From the physical point of view, however, the Lorenz model did not seem at the beginning to have any counterpart in the real world, since it was obtained via a strong and unrealistic truncation of more complex equations for fluid dynamics. In 1975, Haken [6] showed that an isomorphism exists between the Lorenz equations and the equations describing a unidirectional, single-mode, homogeneously broadened, resonantly tuned, two-level laser - conceptually the simplest and most fundamental class of laser. Thus, an important manifestation of the Lorenz model in experimental physics seemed to have been found.

In the case of the laser, the variables $x$, $y$ and $(r-z)$ represent, in appropriate units, the laser field amplitude, induced polarization and population inversion, respectively. The parameter $r$ is the laser pump strength (relative to its value at the onset of laser emission) and $\sigma$ and $b$ define the relaxation rates of the intracavity field (i.e., the cavity losses) and of the population inversion, respectively, where both rates are normalised to the polarization relaxation rate. A linear stability analysis of Eqs. 1 shows that the system's non-trivial stationary solution becomes unstable (allowing for complex behaviour such as, for instance, that of Fig. 1) only when the two following conditions are met:

$$
\begin{aligned}
& \sigma>b+1 \\
& r>\sigma(\sigma+b+3) /(\sigma-b-1)
\end{aligned}
$$

The so-called "bad cavity" condition (Eq. 2 ), means that the cavity losses must be larger than the sum of the molecular relaxation rates to allow for a fast decrease of the laser field intensity. Condition (3) defines the instability threshold (or "second laser threshold" - the first is the emission threshold, $r=1$ ) and implies that pumping must be much larger than $r=1$ (the value required for the onset of laser emission) to allow for rapid increases in the laser intensity.

High gain and high losses are the usual requirements for the appearance of unstable behaviour in dissipative systems. Unfortunately, these conditions are not met in most common classes of lasers: high losses imply large values of the first and second laser thresholds and the latter are difficult to attain. Furthermore, in some lasers such as the $\mathrm{CO}_{2}$ laser, $\sigma$ and, above all, of $b$ are very small in magnitude and the laser behaviour becomes simpler than that described in Fig. 1. So the possibility of observing typical Lorenz chaos seemed to be virtually nil.

\section{Dynamic Features of a Lorenzian System}

\section{Local birfurcation:}

- when a system's parameter reaches a certain value - a bifurcation point a qualitative change in the dynamic regimes occurs corresponding to changes in the trajectory in a limited region of phase space (e.g., a stationary solution becomes modulated, etc.).

\section{Homoclinic explosion:}

- upon increasing a parameter, the appearance of a homoclinic orbit in the phase space is followed by the appearance of an infinite set of extremely close orbits, both unstable and stable (a "strange set").

\section{Generalised instability:}

- the co-existence of two or more solutions, stationary or unstable (co-existence of attractors in phase space).

Feigenbaum scenario (period-doubling road to chaos):

- a sequence of local bifurcations in which the time period of a periodic motion is doubled and is then followed by a reverse sequence with increasing noise which leads to chaos.

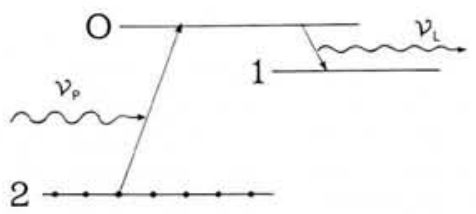

Fig. 2 - Three-level molecular system describing the amplifying medium of an optically pumped laser. Pumping is accomplished by means of a beam of frequency $v_{p}$ (coming from an auxiliary laser) acting on a transition 2-O. The laser radiation of frequency $v_{L}$ is generated on the adjacent transition 0-1. In far-infrared lasers the transition $2-0$ is ro-vibrational $\left(\lambda_{p} \approx 10 \mu \mathrm{m}\right)$ and the transition $0-1$ is rotational $\left(\lambda_{L} \approx\right.$ 50-1000 $\mu \mathrm{m}$ ).

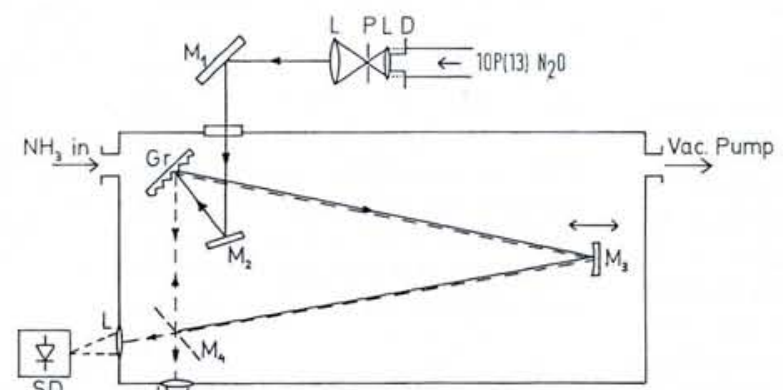

Fig.3 - Optically pumped $\mathrm{NH}_{3}$ ring laser. The $10 \mu \mathrm{m}$ $P(13)$ line of a $\mathrm{N}_{2} \mathrm{O}$ laser is introduced into the cavity through the diaphragm $D$, spatial filter $L P L$, mirrors $M_{1}$ and $M_{2}$, and $10 \mu \mathrm{m}$ grating Gr. Laser radiation at $81 \mu \mathrm{m}$ is generated in the ring cavity composed of the grating $G r$, mirror $\mathrm{SD}$
forward
emission emission 1 th SD bockward by means of the lens $L$ and the Schottky barrier diode SD. The typical operating pressure is $\approx 10 \mathrm{~Pa}$. [From Ref. $2 \mathrm{c}$ ]

\section{Optically Pumped Far-IR Gas Lasers}

However, in 1984, Weiss and Klische [7] suggested that the conditions for Lorenz chaos could be fulfilled in a special class of lasers, namely optically-pumped farinfrared gas lasers. First, in the far infrared, spontaneous emission is much weaker than in the visible spectral region and molecular relaxation is essentially brought about by collisions occurring in the lowpressure gas medium. Molecular relaxation rates are therefore small and cavity losses need not be very high to fulfill Eq. 2; laser thresholds are also low. Second, optical pumping (i.e., pumping by means of another laser beam which acts on a transition sharing the upper level with the laser transition - see Fig. 2 ) is very selective. It transfers population exclusively to the upper level of the laser transition and not to other neighbouring levels: it is therefore very efficient, and the second laser threshold (Eq. 3 ) is attainable.

\section{$\mathrm{NH}_{3}$ laser experiments}

In 1986 Weiss et al. [2a] proved that their intuition was correct. They pumped a $\mathrm{NH}_{3}$-gas filled cavity by means of a $10 \mu \mathrm{m}$ wavelength beam from an auxiliary $\mathrm{N}_{2} \mathrm{O}$ laser, and $81 \mu \mathrm{m}$ wavelength laser radiation was generated in a rotational transition.

To fulfill the conditions (Eqs. 2, 3) of the Lorenz-Haken model, care was taken to keep the laser beam structure as close as possible to a single-mode unidirectional plane wave. In particular, a ring cavity was used and the beam diameter was kept large (Fig. 3). The observed behaviour was in close qualitative agreement with the 
Lorenz-Haken model predictions. A chaotic series of growing spikes, similar to those of Fig. 1b, were suddenly observed in the laser intensity when the pump beam intensity reached values of about 15 times that corresponding to the first laser thres hold. Moreover, windows of regular periodic behaviour were detected within the chaotic domain, and an inverse Feigenbaum scenario ("road out of chaos" see box and Fig. 4) was found when detuning of the cavity was continuously increased from zero (when detuning is allowed, the variables $x$ and $y$ become complex and Eq. 1 is somewhat more complicated - "complex Lorenz equations" or laser Lorenz equations).

Further experiments in 1988 [2b] showed that the same behaviour occurs with another ammonia laser line of a similar type, namely the $153 \mu \mathrm{m}$ line, when pumping is performed with a $\mathrm{CO}_{2}$ laser beam. In spite of the fact that an accurate quantitative comparison between experiment and theory is difficult to make (several molecular and laser parameters values are not known with precision), recent statistical analyses $[2 \mathrm{c}, \mathrm{d}]$ of the experimental data reveal evidence for features identical to those of the Lorenz model results. In particular, the attractor's symmetry is the same, return maps (in which each value in a recorded time series is plotted against a preceding value using both measured and calculated laser intensities) reveal a com-

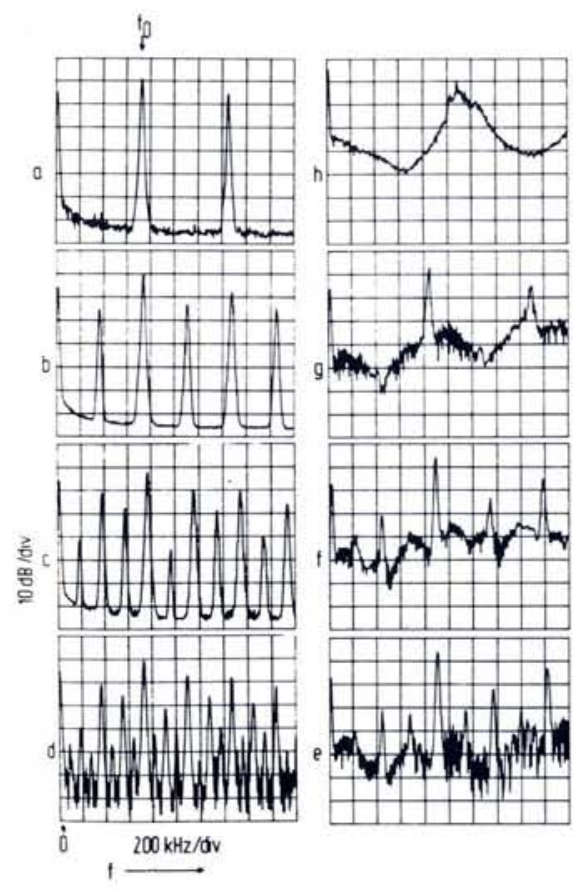

Fig. 4 - Frequency spectra of laser intensity for decreasing cavity detuning (from a to h). A typical Feigenbaum scenario is found: a period-doubling sequence from a to $\mathrm{d}$, followed by a reverse doubling sequence with increasing noise from e to $\mathrm{h}$. In $\mathrm{h}$, the behaviour is chaotic. [From Ref. 2a]
Fig. 5 - Results predicted by the three-level laser model, taking into account the Doppler effect, which are in good agreement with predictions of the Lorenz model.

(a, upper) Time evolution of the laser intensity in the case of chaotic emission.

(b, middle) Bifurcation diagram as a function of the pump field amplitude: the chaotic attractor appears suddenly as $\beta$ increases $\left(C=\right.$ chaotic, $P^{n}=$ periodic with period $n$-times a fundamental period $P$ ).

(c, lower) The same as (b), as a function of the cavity detuning $\Delta^{c}$ : central tuning corresponds to $\Delta^{c} \approx-5$; I.S.N. denotes an inverse sequence of noisy periods. Two typical Feigenbaum roads out of chaos are obtained when $\Delta^{c}$ is varied from central tuning.

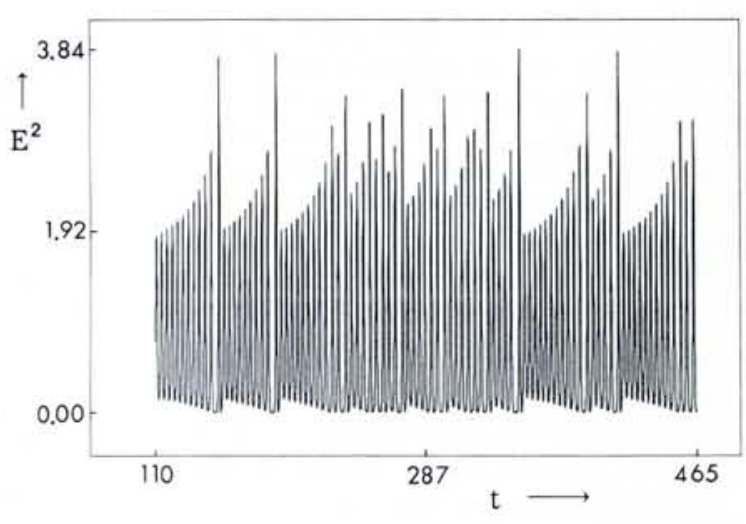

\begin{tabular}{l||c|c|c}
\hline$C$ & $P^{8}$ & $P^{4}$ & $P^{2} \ldots$ \\
\hline & 0.49 & 0.51 & 0.53 \\
\hline
\end{tabular}

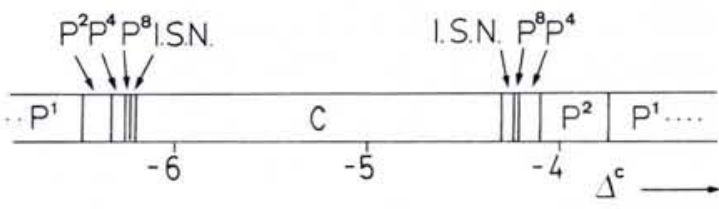

mon structure, and the generalised dimensions are similar (e.g., the "correlation dimension" [1c] is in both cases equal to 2.05 - evidence for the fractal structure of the chaotic attractor).

\section{Additional Effects}

It can be concluded from the experimental results that Lorenz-type behaviour has been observed in a physical system. From the theoretical point of view, however, the data of Weiss et al. raised a basic question. Several groups have argued since 1987 [9-11] that an optically pumped molecular gas laser is actually a far more complex physical system than the simple, homogeneously broadened, twolevel laser described by Eqs. 1. Several effects not taken into account in these equations should play an important rôle in the dynamics of the ammonia laser. One is optical pumping in that pumping is accomplished by means of a coherent field (instead of, for instance, an electrical discharge). This should lead to a transfer of coherence to the molecules and give rise to new effects (so-called three-level effects) such as pump absorption saturation and modulation, Raman pumping, and ac-Stark splitting of the common level 0 described in Fig. 2. Another effect is Doppler broadening where molecules with different velocities in the gas "see" the laser fields with different frequencies owing to the Doppler effect. Yet another is level M-degeneracy where each molecular level (Fig. 2) is really a set of several magnetic sublevels, each of which depends in different ways on the polarization of the laser field. Finally, allowance should be made for the longitudinal and transverse spatial dependence of the pump and generated laser fields along the cavity.

\section{Maintaining Lorenz behaviour}

Unfortunately, all these factors cannot be accounted for together in any solvable laser model. But considering several of them separately leads to interesting results. When only optical pumping is incorporated in the laser model [9], behaviour different to that predicted by the Lorenz model is obtained, thus confirming the important influence of this physical effect. However, when optical pumping and Doppler broadening are both incorporated in the laser model [10], Lorenz-type behaviour is again recovered. Averaging over the different molecular velocities counterbalances or blurs optical pumping effects, leading to significant changes in the effective values of several laser parameters and allowing the appearance of most of the Lorenz features. Figs. 5 and 6 describe results obtained with this three-level model: they include the evolution of the laser intensity (Fig. 5a), bifurcation diagrams (Figs. $5 \mathrm{~b}$ and $\mathrm{c}$ ) and the evolution of the field and phase (Fig. 6), all of which are in close agreement with predictions of the Lorenz-Haken laser model.

It is worth noting that the dynamic evolution of the laser system leads to a phase anholomony (i.e., the phase does not recover its initial value after each period of the dynamic motion - see Fig. 6) similar in several aspects [12] to that of Berry's phase [13]. This resemblance, however, is only formal [14] as the average slope of $\phi(t)$ in Fig. $6 \mathrm{~b}$ depends on the frequency chosen for the representation of the complex laser field.

Recent preliminary results from a model taking into account optical pumping, level $\mathrm{M}$-degeneracy in a simplified way, and polarization of the radiation fields, but ignoring Doppler broadening [11], indicate 
Fig. 6 - Three-level laser model predictions for the case of periodic emission. (a, upper) Time evolution of the laser field amplitude and $(b$, lower) the corresponding field phase $\phi$. Sharp transitions of approximately $\pi$ radians are evidence for the symmetry of the attractor, a two-sided structure similar to that of Fig. 1 a.

that Lorenz-type behaviour is also restored when (as in the ammonia laser experiments) orthogonal linear polarizations for the pump and laser beams are considered.

Even if incomplete, all of these theoretical results suggest that the presence of the additional physical effects increases the number of degrees of freedom of the system but does not increase the dlmensionality of the chaotic attractor. Compensation between the modifications brought about by the factors essentially maintains Lorenz-type features - a result which may provide the basis for understanding the experimentally observed behaviour.

\section{Conclusions}

Experimental and theoretical analyses performed in recent years have shown that an optically pumped far-infrared ammonia laser at wavelengths of 81 and $153 \mu \mathrm{m}$, in spite of its inherent complexity, represents fairly closely the dynamics of the Lorenz model (or, more generally, of the complex Lorenz model).

This result suggests that low-dimensional deterministic Lorenz behaviour demonstrates a structural robustness, or a certain degree of universality, which makes it only slightly sensitive to certain classes of model variations, even if these variations entail a large increase in the number of degrees of freedom of the system.

Some Lorenz-type features have also been found, under particular conditions, in Doppler broadened two-level lasers and in other physical systems including certain electronic circuits. It would be interesting to determine all the possible distinctive structural features and conditions that can make a physical system behave in similar fashion to the Lorenz model. At the same time, the degree of similarity of a given dynamic behaviour to the Lorenz model should be properly quantified, in as much as some differences with respect to this model may remain.

\section{ACKNOWLEDGEMENTS}

The authors are grateful to C.O. Weiss for extensive and fruitful discussions, and to the DGICYT (Spain) for support through projects PB86-0650 and PB89-0319.

\section{REFERENCES}

[1] See, e.g.: a) J. Opt. Soc. Amer. B 2 (1985) 1 and 5 (1988) 875; b) Abraham N.B., Arecchi F.T. and Harrison R.G., Eds. : Instabilities and Chaos in Quantum Optics 11 (Plenum Press) 1988; c) Weiss C.O. and Vilaseca R., Laser Dynamics (VCH) 1991.

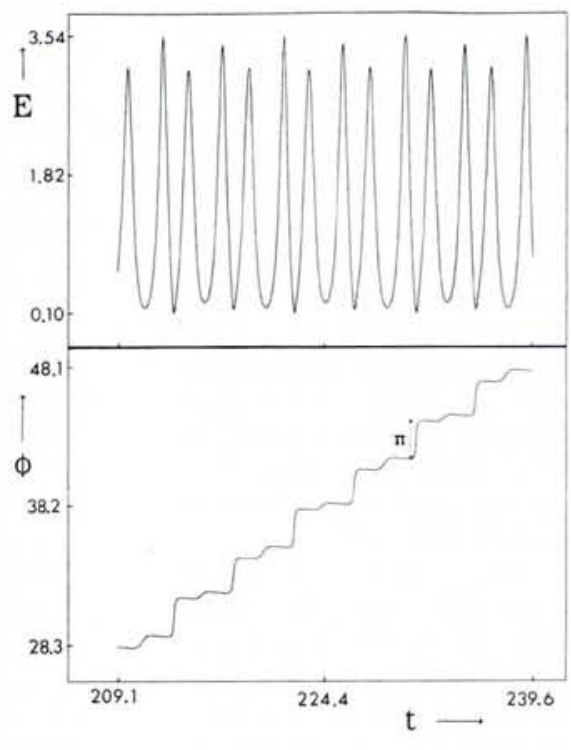

[4] Lugiato L.A., Oldano C. and Narducci L.M., J. Opt. Soc. Am. B 5 (1988) 879; Tamm C. and Weiss C.O., J. Opt. Soc. Am. B 7 (1990) 1043; Coullet P., Gil L. and Rocca F., Optics Comm. 73 (1989) 403.

[5] Sparrow C., The Lorentz Equations: Bifurcations, Chaos and Strange Attractors (Springer-Verlag) 1982.

[6] Haken H., Phys Lett. A 53 (1975) 77.

[7] Weiss C.O. and Klische W., Optics Comm. 51 (1984) 47.

[8] Zeghlache H. and Mandel P., J. Opt. Soc. Am. B 2 (1985) 18; Zeghlache H. et al., Phys Rev. A 38 (1988) 3128.

[9] a) Lawandy N.M. and Ryan J.C., Optics Comm. 63 (1987) 53; b) Khandokhin P.A., Khanin Ya.I. and Koryukin V., Optics Comm. 65 (1988) 367; c) Pujol J. et al., J. Opt. Soc. Am. B 5 (1988) 1004; d) Moloney J.V. et al., Phys. Rev. A. 39 (1989) 1277.

[10] Corbalán R. et al., Optics Comm. 71 (1989) 290; de Valcárel G.J. et al., J. Opt. Soc. Am. B (to be published).

2] a) Weiss C.O. and Brock J., Phys, Rev. Lett. 57 (1986) 2804; b) Weiss C.O., Abraham N.B. and Hübner U., Phys. Rev. Lett. 61 (1988) 1587 ; c) Hübner U., Abraham N.B. and Weiss C.O., Phys. Rev. A 40 (1989) 6354; d) Li M.Y. et al., Optics Comm. 80 (1990) 119.

[3] Arecchi F.T., Meucci R. and Gadomski W., Phys. Rev. Lett. 58 (1987) 2205; Dangoisse D. et al., Europhys. Lett. 6 (1988) 335; Hennequin D. et al., Phys. Rev. A 37 (1988) 2243
[11] Arjona M. et al., Phys Rev. A 41 (1990) 6559.

[12] a) Vilaseca R., de Valcárcel G.J. and Roldán E., Phys. Rev. A 41 (1990) 5269; b) Ning C.Z. and Haken H., Z. Phys. B 81 (1990) 457.

[13] Berry M.V., Proc. R. Soc. London Ser. A 392 (1984) 45; Nature 326 (1987) 277.

[14] Mandel P. et al., Optics Comm. 80 (1991) 262.

Am Institut für Physik der

\section{UNIVERSITÄT BASEL}

ist eine

\section{PROFESSEUR FÜR EXPERIMENTALPHYSIK}

(Ordinariat, Full Professor)

wiederzubesetzen. Bevorzugtes Forschungsgebiet: Moderne Aspekte in Kern- und Teilchenphysik mit Beschleunigern, besonders im Überlappungsbereich der beiden Gebiete.

Von der Kandidatin oder dem Kandidaten wird erwartet: Forschung an führenden europäischen Beschleunigerzentren, Beteiligung an der Lehre auf allen Stufen, gute deutsche Sprachkenntnisse und Bereitschaft zur Mitwirkung an administrativen Aufgaben.

Die üblichen Bewerbungsunterlagen (Lebenslauf, kommentierte Liste der wichtigsten Publikationen, Referenzen) sollten das

\section{Dekanat der Philosophisch-Naturwissenschaftlichen Fakultät Klingelbergstrasse $\mathbf{2 3}$ CH-4056 Basel}

bis 30. März 1992 erreichen. 